Pacific Journal of Mathematics

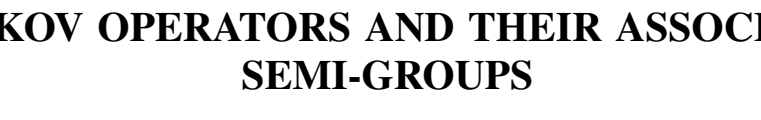




\title{
MARKOV OPERATORS AND THEIR ASSOCIATED SEMI-GROUPS
}

\author{
R. K. Getoor
}

1. Introduction. The present paper is an extension and continuation of our earlier paper "Additive Functionals of a Markov Process" [5] which will be referred to in the sequel as AF. Roughly speaking we consider a temporally homogeneous Markov process, $x(t)$, in a locally compact, separable, metric space and certain other processes derived from it. We always assume $x(t)$ has right continuous paths and we consider processes obtained by stopping $x(t)$ at the boundary of an open set, $G$, and subjecting $x(t)$ to a local "death rate", $V(x)$, in $G$. Our main study is the relationships between the infinitesimal generators of certain semi-groups naturally associated with these processes.

Actually we use a function space approach to stochastic processes and so our results are of an analytic nature (i. e. relations between the transition probabilities and infinitesimal generators) rather than of a measure theoretic nature (i. e. statements above sample functions, etc.). The use of a function space approach simplifies many measure theoretic difficulties associated with conditional probabilities and expectations, but introduces the difficulty that if $G$ is open then $G(t)=\{x(\cdot): x(\tau) \in G$; $0 \leqq \tau \leqq t\}$ is not in general measurable with respect to the $\sigma$-algebra $\mathfrak{B}(\mathfrak{X})$ defined in $\S 2$. It is known [7] that under certain restrictions (implied by our assumptions in $\S 2) G(t)$ is measurable with respect to the appropriate completion of $\mathfrak{B}(\mathfrak{X})$. However, we do not choose to complete $\mathfrak{V}(\mathfrak{X})$ as this introduces the other difficulties mentioned above; instead we consider the set $\{x(\cdot): x(\tau) \in \bar{G} ; 0 \leqq \tau \leqq t\}(\bar{G}$ denotes the closure of $G)$ which is obviously in $\mathfrak{B}(\mathfrak{X})$ and impose a regularity condition on $G$ that insures us that these two sets are roughly the same. (Theorem 2.1 and the ensuing development.)

In $\S 2$ we develop the preliminary machinery that is needed throughout the remainder of the paper. We show in $\S 2$ that all the results of $\mathrm{AF}$ are valid without the assumption $\left(P_{3}\right)$ of $\mathrm{AF}$. In $\S 3$ we investigate the behavior at the boundary of $G$ of the semi-groups introduced in $\S 2$. In $\S 4$ we consider the special case in which the infinitesimal generator of the semi-group associated with $x(t)$ is a local operator. The results of this section also extend and complement those of AF. In the remaining three sections of the paper we study the spectral properties of the semi-groups introduced in the earlier part of the paper.

I would like to take this opportunity to thank Dr. R. M. Blumenthal

Received May 20, 1958. 
for the many enlightening discussions I had with him during the course of this work.

2. Preliminaries. Let $X$ be a locally compact separable metric space with metric $\rho$ and $\mathfrak{B}(X)$ the Borel sets of $X$; that is, the smallest $\sigma$-algebra of subsets of $X$ containing the compact subsets of $X$. Let $\mathfrak{X}$ be the set of all functions from $[0 \leqq t<\infty]$ to $X$ which are right continuous; that is, $x(t) \rightarrow x\left(t_{0}\right)$ as $t \downarrow t_{0}$ for all $t_{0} \geqq 0$. Let $\mathfrak{B}(\mathfrak{X})$ be the $\sigma$ algebra of subsets of $\mathfrak{X}$ generated by sets of the form

$$
\begin{array}{r}
\mathfrak{H}=\left\{x(\cdot) \mid x\left(t_{j}\right) \in A_{j}: 0=t_{0}<t_{1}<\cdots<t_{n} ; A_{j} \in \mathfrak{B}(X),\right. \\
j=0,1, \cdots n\} .
\end{array}
$$

Let $p(t, x, A)$ be a transition probability function defined for $t>0, x \in X$, and $A \in \mathfrak{B}(X)$, such that given an arbitrary probability measure, $\mu$, on $\mathfrak{B}(X)$ there exists a countably additive probability measure, $P_{\mu}$, on $\mathfrak{B}(\mathfrak{X})$ for which

$$
\begin{aligned}
& P_{\mu}(\mathfrak{H})=\int_{A_{0}} \int_{A_{1}} \cdots \int_{A_{n}} \mu\left(d x_{0}\right) p\left(t_{1}, x_{0}, d x_{1}\right) \\
& p\left(t_{2}-t_{1}, x_{1}, d x_{2}\right) \cdots p\left(t_{n}-t_{n-1} x_{n-1}, d x_{n}\right)
\end{aligned}
$$

where $\mathfrak{A}$ is of the form (2.1). If $\mu$ assigns mass one to a single point, $x$, we write $P_{x}$ for $P_{\mu}$.

We make the following assumption throughout the present paper.

$(P)$ There exists a Radon measure, $m$, on $X$ whose support is $X$ and a non-negative function $f(t, x, y)$ defined for $t>0, x \in X, y \in X$ which is jointly measurable (measurablity conditions in $t$ refer to the ordinary Borel sets of $[0 \leqq t<\infty])$ in $(t, x, y)$ such that

$$
p(t, x, A)=\int_{A} f(t, x, y) d m(y) \text { for all } A \in \mathfrak{B}(X) .
$$

To be explicit we assume

$$
\int f(t, x, y) d m(y)=1
$$

for all $t>0, x \in X$ and

$$
f(t+s, x, y)=\int f(t, x, z) f(s, z, y) d m(z)
$$

for all $t, s>0$ and $x, y \in X$. Finally we assume

$$
\int f(t, x, y) d m(x) \leqq M e^{\alpha t}
$$

where $M$ and $\alpha$ are constants independent of $y$ and $\dot{t}$. 
We remark that condition $(\mathrm{P})$ is equivalent to conditions $\left(\mathrm{P}_{1}\right)$ and $\left(\mathrm{P}_{2}\right)$ of $\mathrm{AF}$ and hence the results of $\S 2$ of of $\mathrm{AF}$ are applicable. We intend to use the same notation as in $\mathrm{AF}$ but for the convenience of the reader we repeat the basic definitions. If $A \in \mathfrak{B}(X)$ we define $A_{t}=$ $\{x(\cdot): x(t) \in A\} \in \mathfrak{B}(\mathfrak{X})$. For $\mathfrak{A} \in \mathfrak{B}(\mathfrak{X}), A \in \mathfrak{B}(X), t>0$, and $x \in X$ we define $P(\mathfrak{H} ; t, x, A)=P_{x}\left(\mathfrak{A} \cap A_{t}\right)$. Clearly $P(\cdot ; t, x, A)$ is a finite measure on $\mathfrak{B}(\mathfrak{X})$ for fixed $t, x, A$ and $P(\mathfrak{A} ; t, x, \cdot)$ is a finite measure on $\mathfrak{B}(X)$ for fixed $\mathfrak{A}, t, x$. It was shown in $\mathrm{AF}$ (Theorem 2.1) that $P[\mathfrak{A} ; \cdot, \cdot, A]$ is a measurable function of $(t, x)$ for fixed $\mathfrak{A}, A$. If $\phi[x(\cdot)]$ is a complex valued measurable functional on $\mathfrak{X}$ we write $r[\phi ; t, x, A]$ for the integral of $\phi$ over $\mathfrak{X}$ with respect to the measure $P[\cdot ; t, x, A]$ provided the integral exists. If $\phi \geqq 0$ then $r$ is a measure on $\mathfrak{B}(X)$ for fixed $t, x$ and is measurable in $(t, x)$ for fixed $A$. (AF Theorem 2.3) Finally if $\phi$ is a measurable functional on $\mathfrak{X}$ we denote its integral over $\mathfrak{X}$ with respect to the measure $P_{x}$ by $E\{\phi \mid x(0)=x\}$.

If $A$ is any set let $I[A ; \cdot]$ (or $I_{A}$ ) denote its characteristic function.

Lemma 2.1. If $A \in \mathfrak{B}(X)$ then $I\left[A_{t} ; x(\cdot)\right]$ is jointly measurable in $(t, x(\cdot))$ where $A_{t}$ was defined above.

Proof. We first consider the case in which $A$ is open, thus if $G$ is an arbitrary open set we define $F(t, x(\cdot))=I\left[G_{t} ; x(\cdot)\right]$. Since $F$ only takes on two values, namely 0 and 1 , to show $F$ is jointly measurable in $(t, x(\cdot))$ it is sufficient to show that

$$
\Lambda=\{(t, x(\cdot)) \mid F(t, x(\cdot))=1\} \in \mathfrak{B} \times \mathfrak{B}(\mathfrak{X})
$$

where $\mathfrak{B}$ is the $\sigma$-algebra of the ordinary Borel sets on $[0, \infty)$. Let $\left\{t_{j}^{(n)}\right\}=\left\{j / 2^{n}\right\}$ where $n=1,2, \cdots$ and $j=0,1,2, \cdots$ and define

$$
\mathfrak{A}_{j}^{(n)}=\left\{x(\cdot): F\left((j+1) / 2^{n}, x(\cdot)\right)=1\right\}
$$

and

$$
I_{j}^{(n)}=\left\{t: j / 2^{n} \leqq t<(j+1) / 2^{n}\right\} .
$$

Clearly $\mathfrak{A}_{j}^{(n)} \in \mathfrak{B}(\mathfrak{X})$ and $I_{j}^{(n)} \in \mathfrak{B}$, and using the right continuity of the paths $x(\cdot)$ and the fact that $G$ is open it follows that

$$
\Lambda=\bigcup_{k=1}^{\infty} \bigcap_{n \geqq k} \bigcup_{j=0}^{\infty}\left(I_{j}^{(n)} \times \mathfrak{U}_{j}^{(n)}\right)
$$

and hence $F$ is jointly measurable.

Moreover since $I\left[A_{t} ; x(\cdot)\right]=1-I\left[\left(A^{\prime}\right)_{t} ; x(\cdot)\right]$ where $A^{\prime}$ denotes the complement of $A$, and $I\left[(A \cap B)_{t} ; x(\cdot)\right]=I\left[A_{t} ; x(\cdot)\right] I\left[B_{t} ; x(\cdot)\right]$ it follows that $I\left(A_{t} ; x(\cdot)\right]$ is jointly measurable if $A$ is open or closed and the class of sets for which the lemma holds is closed under finite intersections and 
unions. This class of sets is clearly monotone and thus the lemma follows.

We now introduce some notation that we will use throughout the paper. Let $G$ be an open set in $X$ and let $\bar{G}$ be its closure then for $0 \leqq t<s$ we define

$$
G(t, s)=\{x(\cdot): x(\tau) \in \bar{G} \text {; for all } \tau \text { such that } t \leqq \tau \leqq s\}
$$

and let $G(t)=G(0, t)$. Since $\bar{G}$ is closed and each $x(\cdot)$ is right continuous it follows that $G(t, s) \in \mathfrak{B}(\mathfrak{X})$. We further note that the sets $G(t)$ are increasing as $t$ decreases and hence we define $G(t+)=\cup G(s)$. Let $\mathfrak{B}(t, s)$ be the $\sigma$-algebra of subsets of $\mathfrak{X}$ generated by sets of the form $\left\{x(\cdot): x\left(t_{0}\right) \in A ; t \leqq t_{0} \leqq s\right\}$ and $\mathfrak{B}(t)=\mathfrak{B}(0, t)$. Since the $\sigma$-algebras $\mathfrak{B}(t)$ are decreasing as $t$ decreased we define $\mathfrak{B}(t+)=\cap \mathfrak{B}$. Clearly $G(t, s) \in$ $\mathfrak{B}(t, s)$ for each pair $0 \leqq t<s$ and $G(t+) \in \mathfrak{B}(t \stackrel{s>t}{+})$.

LEMma 2.2. Let $G$ be an open set then the function $I[G(t, s) ; x(\cdot)]$ is jointly measurable in $(t, s, x(\cdot))$.

Proof. We introduce the set $G(t, s-)=\{x(\cdot): x(\tau) \in \bar{G}$; for all $\tau$ such that $t \leqq \tau<s\}$ and clearly $G(t, s-) \in \mathfrak{B}(\mathfrak{X})$. Moreover $I[G(t, s)$;


to prove $I[G(t, s-) ; x(\cdot)]$ jointly measurable in $(t, s, x(\cdot))$. Let $\left\{t_{j}^{(n)}\right\}$ be as in the proof of Lemma 2.1 then for each $n$ we defind $F_{n}(t, s, x(\cdot)=$ $I\left[G\left(t_{j+1}^{(n)}, t_{k}^{(n)}-\right) ; x(\cdot)\right]$ if $t_{j}^{(n)} \leqq t<t_{j+1}^{n}, t_{k}^{(n)} \leqq s<t_{k+1}^{(n)}$ and $k>j$; while if $k=j$ (the only other possibility since $t<s$ ) we define $F_{n}(t, s, x(\cdot))=0$. Clearly each $F_{n}$ is jointly measurable in $(t, s, x(\cdot))$ and using the fact that each $x(\cdot)$ is right continuous it is easy to see that $F_{n}(t, s, x(\cdot)) \rightarrow$ $I[G(t, s-) ; x(\cdot)]$ as $n \rightarrow \infty$. This establishes Lemma 2.2.

Let $G$ be an open subset of $X$ and $V$ a non-negative measurable function on $X$. We define the functional.

$$
\phi[t, x(\cdot)]=\exp \left[-\int_{0}^{t} V[x(\tau)] d \tau\right] I[G(t) ; x(\cdot)]
$$

for each $t>0$ and $x(\cdot) \in \mathfrak{X}$. From Lemma 2.2 and Theorem 3.1 of (AF) it follows that $\phi$ is jointly measurable in its variables and moreover it is clear that $\phi[t, \cdot]$ is measurable with respect to $\mathfrak{B}(t)$ for fixed $t$. We define

$$
\mathrm{K}(V, G ; t, x, A)=r[\phi[t, x(\cdot)] ; t, x, A]
$$

that is the integral of $\phi[t, \cdot]$ with respect to the measure $P[\cdot ; t, x, A]$. This integral certainly exists since the integrand is measurable, nonnegative, and bounded by one. Theorem 2.5 of (AF) implies that 
$K(V, G ; \cdot, \cdot, A)$ is jointly measurable in $(t, x)$ and as before $K(V, G ; t, x, \cdot)$ is a measure on $\mathfrak{B}(X)$.

LEMMA 2.3.

$$
\int K(V, G ; t, x, d y) K(V, G ; s, y, A)=K(V, G ; t+s, x, A) .
$$

Proof. Let $S_{t}$ be the mapping from $\mathfrak{X}$ into $\mathfrak{X}$ defined by $S_{t} x(\cdot)=$ $x(t+\cdot)$ and let $S_{t} \phi[x(\cdot)]=\phi\left[S_{t} x(\cdot)\right]$ for any functional $\phi$, then Lemma 2.3 is an immediate consequence of Theorem 2.4 of (AF) provided we show that $\phi[t, x(\cdot)] S_{t} \phi[s, x(\cdot)]=\phi[t+s, x(\cdot)]$. But

$$
\begin{aligned}
S_{t} \phi[s, x(\cdot)] & =S_{t}\left\{\exp \left[-\int_{0}^{s} V[x(\tau)] d \tau\right] I[G(s) ; x(\cdot)]\right\} \\
& =\exp \left[\int_{t}^{t+s} V[x(\tau)] d \tau\right] I\left[G(s) ; S_{t} x(\cdot)\right] .
\end{aligned}
$$

Moreover $I\left[G(s) ; S_{t} x(\cdot)\right]=1$ if and only if $S_{t} x(\cdot) \in G(s)$, or equivalently $x(\cdot) \in S_{t}^{-1} G(s)=G(t, t+s)$. Thus $S_{t} \phi[s, x(\cdot)]=\exp \left[-\int_{t}^{t+s} V[x(\tau)] d \tau\right]$ $I[G(t, t+s) ; x(\cdot)]$ and the desired result is now obvious.

Of particular importance is the case $V=0$ and we write $K_{G}(t, x, A)$ for $K(0, G ; t, x, A)$. Clearly

$$
0 \leqq K(V, G ; t, x, A) \leqq K_{G}(t, x, A) \leqq p(t, x, A) .
$$

We next show that $G$ is the relevant set in considering $K$.

Lemma 2.4. (i) $K(V, G ; t, x, A)=0$ if $x \notin \bar{G}$.

(ii) $K(V, G ; t, x, A)=K(V, G ; t, x, A \cap \bar{G})$.

Proof. (i) In light of (2.11) it is sufficient to show this for $K_{G}$. For any arbitrary set $\mathfrak{A} \in \mathfrak{B}(\mathfrak{X})$ let $\mathfrak{X}^{0}$ be the set of values of $x(0)$ as $x(\cdot)$ ranges over $\mathfrak{A}$, then $P_{x}[\mathfrak{A}]=0$ if $x \notin \mathfrak{A}^{0}$. Moreover $K_{G}(t, x, A)=$ $r[I[G(t) ; \cdot] ; t, x, A]=P[G(t) ; t, x, A]=P_{x}\left[G(t) \cap A_{t}\right]=0$ if $x \notin \bar{G}$ since $x \notin \bar{G}$ implies $x \notin\left(G(t) \cap A_{t}\right)^{0}$.

(ii) Again it is sufficient to consider the case $K_{G}$. As above $K_{G}(t, x, A)=P_{x}\left[G(t) \cap A_{t}\right]$ but $G(t) \cap A_{t}=G(t) \cap(A \cap \bar{G})_{t}$ and thus $K_{G}(t, x, A)=P_{x}\left[G(t) \cap(A \cap \bar{G})_{t}\right]=K_{G}(t, x, A \cap \bar{G})$.

Lemma 2.4 states that the support of the measure $K(V, G ; t, x, \cdot)$ is contained in $\bar{G}$ and that the support of the function $K(V, G ; t, \cdot, A)$ is contained in $\bar{G}$, thus we can write Lemma 2.3 as

$$
\int_{\bar{G}} K(V, G ; t, x, d y) K(V, G ; s, y, A)=K(V, G ; t+s, x, A) .
$$


In the sequel we will consider $K$ as only being defined for $x \in \bar{G}$ and $A \subset \bar{G}$.

We next introduce the transformations that are the main object of this paper:

$$
\begin{gathered}
\left(U_{t} \varphi\right)(x)=\int \phi(y) p(t, x, d y)=\int \phi(y) f(t, x, y) d m(y) \\
T_{t}[V, G] \phi(x)=\int_{\bar{G}} \varphi(y) K(V, G ; t, x, d y)
\end{gathered}
$$

We will write $T_{t}$ instead of $T_{t}[V, G]$ if there is no chance of confusion. In view of assumption $(P),(2.12)$, and Theorem 3.3 of $\mathrm{AF}$ it follows that $\left\{U_{t} ; t>0\right\}$ and $\left\{T_{t} ; t>0\right\}$ are semi-groups of bounded operators on $L_{2}(X, m)$ and $L_{2}(\bar{G}, m)$ respectively. Moreover

$$
\left\|T_{t}\right\|^{2} \leqq\left\|U_{t}\right\|^{2} \leqq M e^{\alpha t}
$$

We wish to prove that these semi-groups are strongly continuous for $t \geqq 0$ (with $T_{0}=U_{0}=I$ ). In order to do this we introduce two conditions which we will assume throughout the remainder of this paper. The first of these is merely a regularity condition on $G$; if $\partial G$ denotes the boundary of $G$, we assume

$$
m(\partial G)=0 .
$$

The second condition is an assumption on the size of $V$ on $G$, explicitly we assume

$$
\lim _{t \downarrow 0} \int_{0}^{t} \int_{G} f(\tau, x, y) V(y) d m(y) d \tau=0
$$

for almost all $x$ in $G$. We remark that $\left(R_{2}\right)$ is certainly satisfied if for some $\varepsilon>0$ we have $\int_{0}^{\varepsilon} \int_{G} f(\tau, x, y) V(y) d m(y) d \tau<\infty$ for almost all $x$ in $G$. We now state and prove the main theorem of this section $\left(T_{0}=U_{0}=I\right)$.

THEOREM 2.1. The semi-group $\left\{U_{t} ; t \geqq 0\right\}$ is strongly continuous for $t \geqq 0$ and if $\left(R_{1}\right)$ and $\left(R_{2}\right)$ hold then the semi-group $\left\{T_{t}[V, G] ; t \geqq 0\right\}$ is strongly continuous for $t \geqq 0$.

Proof. We prove the theorem for $\left\{T_{t}[V, G] ; t \geqq 0\right\}$ as the result for $\left\{U_{t} ; t \geqq 0\right\}$ is a special case (take $V=0$ and $G=X$, clearly $\left(R_{1}\right)$ and $\left(R_{2}\right)$ are satisfied with this choice of $V$ and $G)$. We write $T_{t}$ for $T_{t}[V, G]$. As


weakly as $t \rightarrow 0$ for all $\phi \in L_{2}(G)$. Suppose $\phi$ is continuous with compact support then using Theorem 2.3 of $A F$ we see that 


$$
\begin{aligned}
\left(T_{t} \varphi\right)(x) & =\int \rho(y) K(V, G ; t, x, d y) \\
= & E\left\{\rho(x(t)) \exp \left(-\int_{0}^{\iota} V[x(\tau)] d \tau\right) I[G(t) ; \cdot] \mid x(0)=x\right\}
\end{aligned}
$$

Clearly $\varphi(x(t)) \rightarrow \varphi(x(0))$ as $t \downarrow 0$ and $I[G(t) ; x(\cdot)] \rightarrow I\left[G\left(0^{+}\right) ; x(\cdot)\right]$ as $t \downarrow 0$ for all $x(\cdot)$.

We next investigate the behavior of the exponential in (2.16). In (2.16) there is no loss of generality in considering $V \equiv 0$ on the complement of $\bar{G}$. With this simplification, since the integrand is non-negative and jointly measurable in $\tau$ and $x(\cdot)$, we obtain

$$
\begin{gathered}
E\left\{\int_{0}^{t} V[x(\tau)] d \tau \mid x(0)=x\right\}=\int_{0}^{t} E\{V[x(\tau)] \mid x(0)=x\} d \tau \\
=\int_{0}^{t} \int_{\bar{G}} f(\tau, x, y) V(y) d m(y) d \tau .
\end{gathered}
$$

Using $\left(\mathrm{R}_{1}\right)$ and $\left(\mathrm{R}_{2}\right)$ it now follows that there exists an $m$-null set $S \subset G$ such that $\int_{0}^{t} V[x(\tau)] d \tau \rightarrow 0$ as a function of $x(\cdot)$ in $P_{x}$ measure provided $x \in G-S$ as $t \rightarrow 0$. Finally the monotoneity of the integral implies that $\int_{0}^{t} V[x(\tau)] d \tau \rightarrow 0$ as $t \rightarrow 0$ for almost all $x(\cdot)$ relative to $P_{x}$ measure if $x \in G-S$ (the exceptional set will, of course, depend on $x$ ). Thus for $x \varepsilon G-S$ the exponential in (2.16) approaches one as $t \downarrow 0$ for almost all $x(\cdot)$ with respect to $P_{x}$ measure. Hence if $x \in G-S$ we obtain using the bounded convergence theorem

$$
\left(T_{t} \varphi\right)(x) \rightarrow E\{\varphi(x(0)) I[G(0+) ; x(\cdot)] \mid x(0)=x\}
$$

as $t \downarrow 0$. For such $x$ the right continuity of $x(\cdot)$ implies that the right hand side of (2.17) reduces to $\phi(x)$. Since $m(S)=m(\partial G)=0$ we obtain that $\left(T_{t} \varphi\right)(x) \rightarrow \varphi(x)$ almost everywhere on $\bar{G}$ as $t \downarrow 0$.

But $\left|\left(T_{t} \varphi\right)(x)\right| \leqq \sup |\varphi(x)|$ and thus if $\psi$ is continuous with compact support it follows that $\left(\psi, T_{t} \varphi\right) \rightarrow(\psi, \varphi)$ as $t \downarrow 0$. Now using the fact that $\left\|T_{t}\right\|$ is uniformly bounded near $t=0$ we easily deduce that $T_{t} \varphi \rightarrow \varphi$ weakly as $t \downarrow 0$ for all $\varphi \in L_{2}(\bar{G})$. This completes the proof of Theorem 2.1.

As mentioned above we will always assume $\left(R_{1}\right)$ and $\left(R_{2}\right)$ in the sequel. Condition $\left(\mathrm{R}_{1}\right)$ implies that $L_{2}(\bar{G})=L_{2}(G)$ and since $K(V, G, t, x, \partial G) \leqq$ $p(t, x, \partial G)=0$, we can (and will) write integrals over $G$ instead of $\bar{G}$.

Exactly as in $\S 4$ of AF we can derive the Darling-Siegert equations (in $\mathrm{AF}$ these were derived only in the case $G=X$ but the method carries over without any trouble) which are for bounded $V$ 


$$
\begin{aligned}
& K(V, G ; t, x, A)=K_{G}(t, x, A) \\
& \quad-\int_{0}^{t} d s \int_{G} V(y) K(V, G ; t-s, y, A) K_{G}(s, x, d y) \\
& K(V, G ; t, x, A)=K_{G}(t, x, A) \\
& \quad-\int_{0}^{t} d s \int_{G} V(y) K_{G}(t-s, y, A) K(V, G ; s, x, d y) .
\end{aligned}
$$

If $\Omega_{G}$ and $\Omega_{G}^{\prime}$ denote the infinitesimal generators of $\left\{T_{t}[0, G] ; t \geqq 0\right\}$ and $\left\{T_{t}[V, G] ; t \geqq 0\right\}$ respectively it then follows exactly as in AF that

$$
\Omega_{G}^{\prime}=\Omega_{G}-V
$$

provided $V$ is essentially bounded on $G$. Moreover if $V$ is not bounded one can show (Theorem 5.2 of AF) that $\mathscr{D}\left(\Omega_{G}\right) \cap \mathfrak{D}(V) \subset \mathscr{D}\left(\Omega_{G}^{\prime}\right)$ and for $\varphi \in \mathfrak{D}\left(\Omega_{G}\right) \cap \mathfrak{D}(V)$ we have $\Omega_{G}^{\prime} \varphi=\left(\Omega_{G}-V\right) \varphi$, where $\mathfrak{D}(\Omega)$ is the domain of $\Omega$ for any operator $\Omega$ and $\mathfrak{D}(V)$ is the domain of $V$ considered as a multiplication operator on $L_{2}(G)$. In the case $G=X$ we will write $\Omega$ and $\Omega^{\prime}$ instead of $\Omega_{X}$ and $\Omega_{X}^{\prime}$.

The proof of Theorem 5.2 in AF depends only on the strong continuity for $t \geqq 0$ of the semi-groups involved. (The use of condition $\left(P_{3}\right)$ in that proof is easily avoided; see the proof of Theorem 4.1 of the present paper.) However, in order to insure the strong continuity of $\left\{T_{t}[V, G] ; t \geqq 0\right\}$ one needs to assume some condition such as $\left(R_{2}\right)$. This isn't done explicitly in the hypothesis of Theorem 5.2 in AF and thus the result is only valid if $V$ satisfies some condition such as $\left(R_{2}\right)$.

In the remainder of this paper integrals involving $K$ in which the region of integration is not specified are understood to be over $G$.

3. Behavior near the boundary. In this section we investigate the behavior of the semi-groups $\left\{T_{t} ; t \geqq 0\right\}$ near the boundary of $G$. Our approach is a straightforward generalization of the methods of [9] p. 308-309 and hence we just sketch the development omitting proofs. However, in many examples it is necessary to use the results of this section in order to identify the operators we have constructed with certain classical operators.

We would like to prove that $T_{t}[V, G] \rho(x) \rightarrow 0$ as $x \rightarrow \partial G$ at least for a fairly wide class of $\phi$ 's. However, even in classical potential theory such statements holds only modulo certain exceptional sets on $\partial G$. Hence we formulate the concept of a regular point on $\partial G$ relative to the process defined by $p(t, x, A)$. We introduce the notation $S\left(x_{0}, \varepsilon\right)=\left\{x \mid \rho\left(x, x_{0}\right)<\varepsilon\right\}$ where $\rho$ is the metric in $\mathrm{X}$.

Definition. Let $G$ be an open set and let $x_{0} \in \partial G$, then $x_{0}$ is called regular provided that there exist positive numbers $\varepsilon=\varepsilon\left(x_{0}\right), \delta=\delta\left(x_{0}\right)$ 
and an open set $C=C\left(x_{0}\right)$ such that

(i ) $C \subset S\left(x_{0}, \delta\right)=S$,

(ii) $C$ and $\bar{G}$ are disjoint,

(iii) $\bar{C} \cap \bar{G}=\left\{x_{0}\right\}$, and

(iv) $p\left(t, x_{0}, C\right) \geqq \varepsilon p\left(t, x_{0}, S\right)$ for all sufficiently small $t$.

Using the general form of the zero-one law given in [2] the methods of [9] p. 308-309 are easily modified to yield the following theorem.

THEOREM 3.1. Let $G$ be an open set and let $x_{0} \in \hat{o} G$ be regular. Let $\int \varphi(y) f(t, x, y) d m(y)$ be a bounded continuous function on $X$ whenever $\varphi$ is a bounded continuous function on $X$. Under these conditions

$$
\lim _{x \rightarrow x_{0}} K(V, G ; t, x, A)=0,
$$

and if $\varphi$ is a bounded continuous function on $G$ then

$$
\lim _{x \rightarrow x_{0}} \int \varphi(y) K(V, G ; t, x, d y)=0 .
$$

4. Local Operators. In $\S 2$ we introduced the infinitesimal generators $\Omega$ and $\Omega_{G}$ of $\left\{U_{t} ; t \geqq 0\right\}$ and $\left\{T_{t}[0, G] ; t \geqq 0\right\}$ respectively. We intend to call $\Omega_{G}$ the generalized restriction of $\Omega$ to $G$. The purpose of this section is to show that if $\Omega$ is a local operator (to be defined shortly) then $\Omega_{G}$ is the ordinary restriction of $\Omega$ to $G$. In order to attack this problem we first establish an approximation theorem which will also be of use in $\S 6$.

In general $L_{2}(G)$ can be imbedded in $L_{2}(X)$ by the map

$$
U: L_{2}(G) \rightarrow L_{2}(X)
$$

where

$$
(U \varphi)(x)=\left\{\begin{array}{rl}
\varphi(x) & x \in G \\
0 & x \notin G
\end{array} .\right.
$$

Thus $L_{2}(G)$ can be regarded as a closed subspace of $L_{2}(X)$ and in the sequel we will adopt this point of view. We define functions $V_{n}$ as follows

$$
V_{n}(x)=\left\{\begin{array}{l}
0 x \in \bar{G} \\
n x \notin \bar{G}
\end{array}\right.
$$

and we let $\left\{T_{t}^{(n)} ; t \geqq 0\right\}$ be the semi-group $\left\{T_{t}\left[V_{n}, X\right] ; t \geqq 0\right\}$, and $\left\{T_{t} ; t \geqq 0\right\}$ the semi-group $\left\{T_{t}[0, G] ; t \geqq 0\right\}$. Our approximation theorem can now be stated as follows. 
Theorem 4.1. For all $\phi \in L_{2}(G)$ and each $t>0$ we have

$$
\lim T_{l}^{(n)} \mathscr{\rho}=T_{t} \rho
$$

where the limit is taken in $L_{2}(X)$.

Proof. Let

$$
K_{n}(t, x, A)=K\left(V_{n}, X ; t, x, A\right)=r\left[\exp \left(-\int_{0}^{t} V_{n}[x(\tau)] d \tau\right) ; t, x, A\right] .
$$

Using the right continuity of $x(\cdot)$ we see that $\lim _{n \rightarrow \infty} \exp \left(-\int_{0}^{t} V_{n}[x(\tau)] d \tau\right)=$ $I[G(t-) ; x(\cdot)]$, where $G(t-)=\{x(\cdot): x(\tau) \in G ; 0 \leqq \tau<t\}$. Thus by the monotone convergence theorem we obtain $\lim K_{n}(t, x, A)=P[G(t-) ; t, x ; A]$ or if $A \subset \bar{G}$ that $K_{n}(t, x, A) \downarrow K_{G}(t, x, A)$ as $n \rightarrow \infty$. It now follows easily that if $\rho \in L_{2}(G)$ and is continuous with compact support then $\left(T_{t}^{(n)} \varphi\right)(x) \rightarrow\left(T_{t} \varphi\right)(x)$ pointwise as $n \rightarrow \infty$. (See proof of Theorem 5.2 in AF.) But

$$
\left|\left(T_{t}^{(n)} \varphi\right)(x)\right| \leqq \int|\varphi(y)| K_{n}(t, x, d y) \leqq\left(T_{t}^{(1)}|\varphi|\right)(x)
$$

which is in $L_{2}(X)$ and hence by the Lebesgue bounded convergence theorem $\left\|T_{t}^{(n)} \varphi-T_{t} \varphi\right\| \rightarrow 0$ if $\varphi$ is continuous with compact support. This implies (4.1) since $\left\|T_{t}^{(n)}\right\|$ is uniformly bounded in $n$.

We now define the concept of a local operator in $L_{2}(X)$. All operators are assumed linear.

Definition. An operator, $\Omega$, in $L_{2}(X)$ will be called a local operator if whenever $\mathcal{P} \in \mathscr{D}(\Omega)$ and $G$ is any open set with $m(\partial G)=0$ then $\varphi I_{G} \in \mathscr{D}(\Omega)$ and $I_{G} \Omega \mathscr{P}=\Omega\left(I_{G} \varphi\right)$ as elements of $L_{2}(X)^{1}$.

The following properties of local operators are immediate. $L I$ If $\varphi=0$ a. e. on an open set, $G$, with $m(\partial G)=0$ and $\varphi \in \mathscr{D}(\Omega)$ then $\Omega \varphi=0$ a. e. on $G$.

$L 2$ If $f, g \in \mathscr{D}(\Omega)$ and $f=g$ a.e. on an open set, $G$, with $m(\partial G)=0$ then $\Omega f=\Omega g$ a. e. on $G$.

$L 3 \Omega$ is a local operator if and only if for all open sets $G$ with $m(\partial G)=0$ we have $I_{G} \Omega=\Omega I_{G}$ where $I_{G}$ is being considered as a multiplication operator on $L_{2}(X)$.

1 The condition $m(\partial G)=0$ is needed if differential operators in $E^{n}$ are to be local operators under our definition. 
If $\Omega$ is a local operator and $G$ an open set with $m(\partial G)=0$, then the restriction, $\hat{\Omega}_{G}$, of $\Omega$ to $G$ is an operator in $L_{2}(G)$ which is defined as follows (we regard $L_{2}(G)$ as a subspace of $L_{2}(X)$ ):

$$
\begin{gathered}
\mathscr{D}\left(\hat{\Omega}_{G}\right)=\left\{\phi: \varphi \in L_{2}(G) \text { and } \varphi \in \mathscr{Q}(\Omega)\right\} \text { and for } \varphi \in \mathscr{D}\left(\hat{\Omega}_{G}\right) \\
\hat{\Omega}_{G} \varphi=I_{G} \Omega \varphi \in L_{2}(G) .
\end{gathered}
$$

Note that since $\Omega$ is local (4.2) can be written $\hat{\Omega}_{G} \mathcal{P}=\Omega\left(I_{G} \mathscr{T}\right)=\Omega \Phi$ since $\phi \in L_{2}(G)$ implies $I_{G} \varphi=\varphi$ as elements of $L_{2}(X)$.

We now state and prove the main theorem of the present section.

TheOREM 4.2. Let $\Omega$ and $\Omega_{G}$ be the infinitesimal generators of $\left\{U_{t}\right.$; $t \geqq 0\}$ and $\left\{T_{t}[0, G] ; t \geqq 0\right\}$ respectively, then if $\Omega$ is a local operator we have $\Omega_{G}=\hat{\Omega}_{G}$, that is, the generalized restriction of $\Omega$ to $G$ equals the ordinary restriction of $\Omega$ to $G$ if $\Omega$ is a local operator. Of course, we are assuming $m(\partial G)=0$.

Proof. Let $T_{t}^{(n)}=T_{t}\left[V_{n}, X\right]$ be the semi-groups constructed in Theorem 4.1 and from Theorem 4.1 we know that $T_{t}^{(n)} \mathscr{P} \rightarrow T_{t} \mathcal{P}=T_{t}[0, G]$ $\phi$ for all $\phi \in L_{2}(G)$. Let $I_{\lambda}, J_{\lambda}$, and $J_{\lambda}^{(n)}$ be the resolvents of the semigroups $\left\{U_{t} ; t \geqq 0\right\},\left\{T_{t} ; t \geqq 0\right\}$ and $\left\{T_{t}^{(n)} ; t \geqq 0\right\}$ respectively, it then follows from Theorem 4.1 that $J_{\lambda}^{(n)} \mathscr{P} \rightarrow J_{\lambda} \varphi$ for all $\phi \in L_{2}(G)$. Moreover we know from Theorem 5.1 of $\mathrm{AF}$ that the infinitesimal generator of $\left\{T_{t}^{(n)} ; t \geqq 0\right\}$ is $\Omega-V_{n}$. Let $\varphi \in \mathfrak{O}(\Omega)$ then since $\Omega$ is local $I_{G} \varphi \in \mathscr{D}\left(\Omega-V_{n}\right)$ for all $n$. We recall the fact that for each fixed $\lambda$ the range of the resolvent of a strongly continuous $(t \geqq 0)$ semi-group is precisely the domain of the infinitesimal generator of the semi-group [6]. Let $\lambda$ be fixed then for each $n$ there exists $\psi_{n} \in L_{2}(X)$ such that

$$
I_{G} \mathcal{P}=J_{\lambda}^{(n)} \psi_{n}
$$

But then

$$
\psi_{n}=\left[\lambda-\left(\Omega-V_{n}\right)\right] I_{G} \varphi=\lambda I_{G} \varphi-\Omega I_{G} \varphi+V_{n} I_{G} \mathscr{P}=I_{G}(\lambda \varphi-\Omega \varphi)
$$

since $V_{n} I_{G}=0$. Hence $\psi_{n}=\psi=I_{G}(\lambda \varphi-\Omega \varphi) \in L_{2}(G)$ and $J_{\lambda}^{(n)} \psi \rightarrow J_{\lambda} \psi$. But this implies that $I_{G} \varphi=J_{\lambda} \psi$ or $I_{G} \varphi \in \mathscr{D}\left(\Omega_{G}\right)$ and

$$
\Omega_{G}\left(I_{G} \varphi\right)=\lambda J_{\lambda} \psi-\psi=\lambda I_{G} \varphi-\left[\lambda I_{G} \varphi-I_{G} \Omega \varphi\right]=\Omega I_{G} \varphi .
$$

Thus if $\varphi \in \mathscr{D}\left(\hat{\Omega}_{G}\right)$ it follows by definition that $\varphi \in \mathscr{D}(\Omega)$ and $\varphi \in L_{2}(G)$, hence $\phi=I_{G} \mathscr{\mathscr { P }} \in \mathscr{D}\left(\Omega_{G}\right)$ and $\hat{\Omega}_{G} \mathscr{P}=\Omega \mathscr{P}=\Omega_{G} \mathscr{P}$, that is $\hat{\Omega}^{G} \subset \Omega_{G}$.

Conversely suppose $\varphi \in \mathscr{D}\left(\Omega_{G}\right)$ then there exists $\psi \in L_{2}(G)$ such that $\phi=$ $J_{\lambda} \psi$. Define $\varphi_{n}=J_{\lambda}^{(n)} \psi \in L_{2}(X)$, then $\varphi_{n} \in \mathscr{D}\left(\Omega-V_{n}\right)$ and $\left[\lambda-\left(\Omega-V_{n}\right)\right] \mathcal{P}_{n}=$ $\psi$. Multiply this last equation by $I_{G}$ and using the facts that $\Omega$ is local 
and $I_{G} V_{n}=0$ we obtain $(\lambda-\Omega) I_{G} \varphi_{n}=I_{G} \psi=\psi$ since $\psi \in L_{2}(G)$. But $\varphi_{n}=J_{\lambda}^{(n)} \psi \rightarrow J_{\lambda} \psi=\varphi$ and hence $I_{G} \varphi_{n} \rightarrow I_{G} \varphi=\varphi$ since $\varphi \in L_{2}(G)$. Combining these results with the fact that $\Omega$ is closed we see that $\varphi \in \mathscr{D}(\Omega)$ and $\Omega \varphi=\lambda \varphi-\psi=\lambda \varphi-\left(\lambda-\Omega_{G}\right) \varphi=\Omega_{G} \varphi$. Since $\varphi \in L_{2}(G)$ this implies $\varphi \in \mathscr{D}\left(\hat{\Omega}_{G}\right)$ and $\hat{\Omega_{G}} \varphi=\Omega \varphi=\Omega_{G} \varphi$. That is $\Omega_{G} \subset \hat{\Omega}_{G}$. This establishes Theorem 4.2.

Let $\Omega^{\prime}$ be the infinitesimal generator of $\left\{T_{t}[V, X] ; t \geqq 0\right\}$ then we know that if $V$ is bounded $\Omega^{\prime}=\Omega-V$. In general if $V$ is not bounded we can only conclude that $\Omega-V \subset \Omega^{\prime}$ (Theorem 5.2 of AF). However, if $\Omega$ is a local operator we can obtain more information about $\Omega^{\prime}$, at least if we assume a mild regularity condition on the underlying space $X$ and the measure $m$. We introduce the following condition which will be referred to as $(\mathrm{R})$ :

(R) There exists a sequence, $\left\{G_{n}\right\}$, of open sets with compact closure such that $X=\cup G_{n}$ and $m\left(\partial G_{n}\right)=0$ for all $n$.

Let $\Omega$ be a local operator in $L_{2}(X)$ and assume $(\mathrm{R})$ holds, then if $I_{G_{n}} \varphi \in \mathscr{D}(\Omega)$ for all $n$ we can define a function, $(\Omega \varphi)(x)$, which is determined almost everywhere but which will not in general be in $L_{2}(X)$. We define $(\Omega \varphi)(x)=\left(\Omega I_{G_{n}} \varphi\right)(x)$ provided $x \in G_{n}$ and since $\Omega$ is local it is clear that $(\Omega \mathscr{P})(x)$ is well defined independently of the particular sequence, $\left\{G_{n}\right\}$, chosen up to a set of measure zero. The point of the matter is that although $I_{G_{n}} \rho \in \mathscr{D}(\Omega)$ for all $n$ it may happen that $\varphi \mathscr{D} \notin(\Omega)$ because $(\Omega \varphi)(\cdot) \notin L_{2}(X)$. This is a familiar phenomenon in the case of the best known local operators, i.e., differential operators in Euclidean spaces. We will say that the function, $V$, is locally bounded if it is essentially bounded on compact sets. The following theorem gives precise information about $\Omega^{\prime}$ in the case that $\Omega$ is local, $V$ is locally bounded, and $(R)$ is satisfied. It should be compared with Theorems 5.1 and 5.2 of $\mathrm{AF}$.

THEOREM 4.3. Let $\Omega$ be a local operator, and $V$ be locally bounded on $G$, and $(\mathrm{R})$ be satisfied, then for every $\varphi \in \mathscr{D}\left(\Omega^{\prime}{ }_{G}\right)$ we have

$$
\left(\Omega_{G}^{\prime} \varphi\right)(x)=(\Omega \varphi)(x)-V(x) \varphi(x)
$$

almost everywhere. However neither $(\Omega \varphi)(\cdot)$ nor $V \varphi$ need be in $L_{2}(G)$.

Proof. We prove the theorem in the case $G=X$, the general result following by a completely analogous argument and an appeal to Theorem 4.2.

Let $\mathscr{D}(V)=\left\{\varphi: \varphi \in L_{2}(X)\right.$ and $\left.V \varphi \in L_{2}(X)\right\}$. Let $\varphi \in \mathscr{D}\left(\Omega^{\prime}\right)$ and $\left\{G_{k}\right\}$ be the sequence of open sets in (R). We first show that $I_{G_{k}} \rho \in$ $\mathscr{D}(\Omega) \cap \mathscr{D}(V)$ for all $k$. If 


$$
V_{n}(x)= \begin{cases}V(x) & \text { if } V(x) \leqq n \\ n & \text { if } V(x)>n\end{cases}
$$

then let $J_{\lambda}^{(n)}$ be the resolvent of the semi-group $\left\{T_{t}\left[V_{n}, X\right] ; t \geqq 0\right\}$ and $J_{\lambda}$ the resolvent of $\left\{T_{t}[V, X] ; t \geqq 0\right\}$. It was shown in AF (during the proof of Theorem 5.2, see also the proof of Theorem 4.1 of the present paper) that $T_{t}\left[V_{n}, X\right] \rho \rightarrow T_{t}[V, X] \rho$ and $J_{\lambda}^{(n)} \mathcal{P} \rightarrow J_{\lambda} \rho$ for all $\varphi \in L_{2}(X)$. Moreover the infinitesimal generator of $\left\{T_{t}\left[V_{n}, X\right] ; t \geqq 0\right\}$ is $\Omega-V_{n}$ while that of $\left\{T_{t}[V, X] ; t \geqq 0\right\}$ is by definition $\Omega^{\prime}$.

Now since $\varphi \in \mathfrak{O}\left(\Omega^{\prime}\right)$ there exists $\psi \in L_{2}(X)$ such that $\varphi=J_{\lambda} \psi$. Define $\varphi_{n}=J_{\lambda}^{(n)} \psi$ and then $\left[\lambda-\left(\Omega-V_{n}\right)\right] \varphi_{n}=\psi$ or $\Omega \varphi_{n}=\lambda \varphi_{n}+V_{n} \varphi-\psi$. Multiplying this last equation by $I_{G_{k}}$ and using the fact that $\Omega$ is local we obtain

$$
\Omega\left(I_{G_{k}} \varphi_{n}\right)=\lambda I_{G_{k}} \rho_{n}+V_{n} I_{G_{k}} \rho_{n}-I_{G_{k}} \Psi .
$$

But $\varphi_{n}=J_{\lambda}^{(n)} \psi \rightarrow J_{\lambda} \psi=\rho$ and hence $I_{G_{k}} \rho_{n} \rightarrow I_{G_{k}} \varphi$. Moreover since $\bar{G}_{k}$ is compact and $V$ is locally bounded it follows that $V_{n} I_{G_{k}} \rho_{n} \rightarrow V I_{G_{k}} \rho$ in $L_{2}$. Combining the fact that $\Omega$ is closed with the above remarks we see that $I_{r_{k}} \rho \in \mathscr{C}(\Omega)$ and hence $I_{r_{h}} \mathscr{P} \in \mathscr{D}(\Omega) \cap \mathscr{D}(V)$.

From (4.5) we obtain

$$
\begin{aligned}
\Omega\left(I_{G_{k}} \rho\right) & =\lambda I_{G_{k}} \rho+V I_{G_{k}} \rho-I_{G_{k}} \psi \\
& =\lambda I_{G_{k}} \rho+V I_{G_{k}} \rho-I_{G_{k}}\left(\lambda-\Omega^{\prime}\right) \varphi \\
& =V I_{G_{k}} \rho+I_{G_{k}} Q^{\prime} \rho .
\end{aligned}
$$

Since $X=\cup G_{k}$ this last equation can be written

$$
\left(\Omega^{\prime} \varphi\right)(x)=(\Omega \varphi)(x)-V(x) \varphi(x)
$$

almost everywhere on $X$, where $(\Omega \varphi)(x)$ is the function defined above. This completes the proof of Theorem 4.3.

5. The Density of K. Since $K(V ; G ; t, x, A) \leqq p(t, x, A)=\int_{A} f(t, x, y)$ $d m(y)$ it is evident that $K$ is absolutely continuous with respect to $m$. The following theorem shows that the density can be chosen to be jointly measurable in its variables.

Theorem 5.1. There exists a function $k(V, G ; t, x, y)=k(t, x, y) d e$ fined for $t>0$ and $(x, y) \in G \times G$ such that $k$ is jointly measurable in all its variables and for fixed $(t, x)$

$$
\begin{aligned}
& K(V, G ; t, x, A)=\int_{A} k(t, x, y) d m(y) \text { for all } A, \text { and } \\
& k(t, x, y) \leqq f(t, x, y) \text { for almost all } y .
\end{aligned}
$$


Proof. In the present proof $V$ and $G$ are fixed and so we will suppress them in our notation. Let $G=\cup S_{n}$ where the $S_{n}$ 's are disjoint measurable sets of finite measure. Since $X$ is a separable metric space $\mathfrak{B}(X)$ is countably generated and $m$ restricted to $S_{n}$ is a finite measure. Under these conditions one can show using a technique due to Doob [3, pp. 343-344] that there exists a function, $k_{n}(t, x, y)$, defined for $t>0$, $x \in G, y \in S_{n}$ such that $k_{n}$ is jointly measurable in $(t, x, y)$ (for this one needs the fact that $K(\cdot, \cdot, A)$ is jointly measurable in $(t, x)$ which was established in $\S 2)$ and $k_{n}(t, x, \cdot)$ is a density for $K(t, x, \cdot)$ with respect to $m$ restricted to $S_{n}$ for each pair $(t, x)$. The details of this construction will be omitted since one merely has to put in $(t, x)$ in the appropriate spots in Doob's construction. (See [1] where the details are give.)

If we define

$$
k(t, x, y)=\sum_{n=1}^{\infty} k_{n}(t, x, y) I\left[S_{n} ; y\right]
$$

it is clear that $k$ has the desired properties.

6. The Spectral Decomposition: Compact case. We begin with the following simple remarks.

THEOREM 6.1. If $f(t, x, y)=f(t, y, x)$ almost everywhere, $m \times m$ then each of the operators $T_{t}[V, G]$ is self-adjoint and $\left\|T_{t}[V, G]\right\| \leqq 1$.

Proof. The fact that $U_{t}$ and $T_{t}[V, X]$ are self-adjoint is contained in the corollary to Theorem 2.5 of AF. Using Theorem 4.1 and the fact that the strong limit of self-adjoint operators is self-adjoint we see that $T_{t}[V, G]$ is self-adjoint. The symmetry of $f$ implies that $M$ and $\alpha$ can be taken to be 1 and 0 respectively in (2.6). Hence (2.15) yields $\| T_{t}[V, G] \mid \leqq 1$.

Theorem 6.2. Suppose $G$ and $f$ are such that

$$
\int_{G} \int_{G} f(t, x, y)^{2} d m(x) d m(y)<\infty,
$$

then each of the operators $T_{t}[V, G]=T_{t}$ is an integral operator of finite double norm ${ }^{2}$. That is

$$
\left(T_{t} \varphi\right)(x)=\int k(t, x, y) \varphi(y) d m(y)
$$

where $k$ is jointly measurable in its variables and

2 The theory of operators of finite double norm (also called Hilbert-Schmidt operators) can be found in [10, sec. 97] and slightly more generally in [12], especially pp. 242-248 and p. 353 , 


$$
\iint k(t, x, y)^{2} d m(x) d m(y)<\infty .
$$

(Integrals involving $k$ are understood to be over $G$ unless otherwise specified.)

Proof. This is an immediate consequence of Theorem 5.1.

THEOREM 6.3. Let $(K)$ of Theorem 6.2 hold and $f(t, x, y)=f(t, y, x)$ a. e., then $T_{t}[V, G]=T_{t}$ is a positive definite, self-adjoint operator of finite double norm. Moreover there exists a sequence $\left\{\lambda_{j}\right\}$ of real numbers such that $0 \leqq \lambda_{1} \leqq \lambda_{2} \leqq \cdots$ with $\lambda_{j} \rightarrow+\infty$, and a complete orthonormal system $\left\{\rho_{j}\right\}$ in $L_{2}(G)$ such that

$$
T_{t} \mathcal{P}_{j}=e^{-\lambda_{j} t} \mathscr{\varphi}_{j} \text { for all } t>0
$$

and

$$
\Omega_{G}^{\prime} \varphi_{j}=-\lambda_{j} \varphi_{j}
$$

where $\Omega_{G}^{\prime}$ is the infinitesimal generator of $\left\{T_{t} ; t \geqq 0\right\}$.

Proof. From Theorem 6.1 it follows that each $T_{t}$ is self-adjoint and hence it is easy to see that $k(t, x, y)=k(t, y, x)$ almost everywhere, $m \times m$ for fixed $t(k(t, x, y)$ is the function defined in Theorem 6.2). Thus $T_{t}$ is given by an integral operator whose kernel is of finite double norm and symmetric.

We next show that each $T_{t}$ is positive definite. Since each $T_{t}$ is self-adjoint and $T_{t}=T_{t / 2} T_{t / 2}$ we see that $\left(T_{t} \varphi, \varphi\right)=\left\|T_{t / 2} \varphi\right\|^{2} \geqq 0$. Suppose $T_{t} \rho=0$, then $\left\|T_{t / 2} \varphi\right\|^{2}=\left(T_{t} \rho, \varphi\right)=0$ or $T_{t / 2} \varphi=0$. Repeating this argument we see that $T_{t / 2} n \varphi=0$ for all $n$ and since the semi-group $\left\{T_{t}: t \geqq 0\right\}$ is strongly continuous it now follows that $\varphi=0$. Thus $T_{t}$ is positive definite.

Since $T_{1}$ is a compact, self-adjoint, positive definite operator it possesses a discrete set of eigen values $\left\{\mu_{j}\right\}^{3}$ with corresponding eigen functions $\phi_{j}$ such that $0<\mu_{j} \leqq\left\|T_{1}\right\| \leqq 1$ and $\mu_{j} \downarrow 0$. Moreover the $\varphi_{j}$ may be taken so that $\left\{\mathscr{\rho}_{j}\right\}$ is a complete orthonormal system in $L_{2}(G)$. Define $\lambda_{j}=-\log \mu_{j}$ then $\lambda_{j} \geqq-\log \left\|T_{1}\right\| \geqq 0$ and $\lambda_{j} \uparrow+\infty$. We now prove that $\phi_{j}$ is an eigen function of $T_{t}$ with eigenvalue $e^{-\lambda_{j} t}$ for each $t>0$. Consider

$$
0=\left(T_{1}-\mu_{j}\right) \varphi_{j}=\left(T_{1 / 2}-\mu_{j}^{1 / 2}\right)\left(T_{1 / 2}+\mu_{j}^{1 / 2}\right) \varphi_{j}
$$

and let

${ }^{3}$ Each distinct $\mu_{j}$ is, of course, repeated according to its (finite) multiplicity. 


$$
\psi=\left(T_{1 / 2}-\mu_{j}^{1 / 2}\right) \mathcal{P}_{j},
$$

then

$$
\left(T_{1 / 2}+\mu_{j}^{1 / 2}\right) \psi=0
$$

Hence

$$
0=\left\|\left(T_{1 / 2}+\ell_{j}^{1 / 2}\right) \Psi\right\|^{2}=\left\|T_{1 / 2} \psi\right\|^{2}+\mu_{j}\|\psi\|^{2}+2 \mu_{j}\left(T_{1 / 2} \psi, \psi^{r}\right) .
$$

But $\mu_{j}>0$ and $T_{1 / 2}$ is positive definite, thus $\psi=0$ or $T_{1 / 2} \varphi_{j}=\mu_{j}^{1 / 2} \varphi_{j}=$ $e^{-1 / 2}{ }_{j} \mathcal{P}_{j}$. Similarly if $t=m / 2^{n}$ we see that $T_{t} \varphi_{j}=e^{-\lambda_{j} t} \mathscr{Q}_{j}$ and using the strong continuity of the semi-group this then holds for all $t>0$. Moreover $\left\{\mathscr{P}_{j}\right\}$ being a complete orthonormal system implies that the numbers $e^{-\lambda_{j} t}$ exhaust the eigen values of $T_{t}$. Finally the fact that $T_{t / 2}$ is of finite double norm implies that

$$
\sum_{j=1}^{\infty} e^{-\lambda_{j} t}<\infty \text { for each } t>0 .
$$

To complete the proof of Theorem 6.3 we must show that each $\rho_{j} \in \mathscr{Y}\left(\Omega_{G}^{\prime}\right)$ and that (6.4) holds. Suppose $T_{t} \mathscr{P}=e^{-\lambda t} \mathscr{P}$ and let $J_{\mu}$ be the resolvent of the semi-group $\left\{T_{t} ; t \geqq 0\right\}$, then

$$
J_{\mu} \rho=\int_{0}^{\infty} e^{-\mu t} T_{t} \varphi d t=\int_{0}^{\infty} e^{-\mu t} e^{-\lambda t} \varphi d t=\frac{1}{\lambda+\mu} \varphi .
$$

Thus $\rho=J_{\mu}[(\lambda+\mu) \varphi]$ which implies that $\rho \in \mathscr{C}\left(\Omega_{G}^{\prime}\right)$. Also

$$
\left(\mu-\Omega_{G}^{\prime}\right) J_{\mu} \rho=\varphi=\left(\mu-\Omega_{G}^{\prime}\right) \frac{1}{\lambda+\mu} \varphi
$$

or $\Omega_{G}^{\prime} \varphi=-\lambda \varphi$. This proves (6.4) and again since $\left\{\varphi_{j}\right\}$ is a complete orthonormal system $\Omega_{G}^{\prime}$ has a discrete spectrum consisting precisely of the numbers $-\lambda_{j}$. This completes the proof of Theorem 6.3.

The $\mathscr{P}_{j}^{\prime} \mathrm{s}$ are, of course, only determined almost everywhere. We now pick the $\mathscr{\rho}_{j}^{\prime}$ s such that

$$
\begin{aligned}
\varphi_{j}(x) & =e^{\lambda_{j}} \int K(V, G ; 1, x, d y) \varphi_{j}(y) \\
& =e^{\lambda_{j}} \int k(1, x, y) \varphi_{j}(y) d m(y)
\end{aligned}
$$

for all $x$, and in the sequel $\varphi_{j}(x)$ will denote these functions which are defined everywhere.

THeOREm 6.4 We assume the same hypotheses as in Theorem 6.3 and that $\varphi_{i}(x)$ is defined by (6.6), then $\sum_{j=1}^{\infty} e^{-\lambda t} \varphi_{j}(x) \varphi_{j}(y)$ converges absolutely for fixed $t>0, x, y$, and 


$$
k(t, x, \cdot)=\sum_{j=1}^{\infty} e^{-\lambda_{j} t} \mathcal{P}_{j}(x) \mathcal{P}_{j}(\cdot)
$$

for almost all y for fixed $(t, x)$.

Proof. We first remark that since

$$
\int K(V, G ; t, x, d z) K(V, G ; s, z, A)=K(V, G ; t+s, x, A)
$$

identically in its variables it easily follows that

$$
k(t+s, x, y)=\int k(t, x, z) k(s, z, y) d m(z)
$$

for all most all $y$ for fixed $(t, s, x)$ where the exceptional set, of course, depends on $(t, s, x)$.

We next show that for all $t>0$ and all $x$

$$
\rho_{j}(x)=e^{\lambda_{j} t} \int k(t, x, y) \varphi_{j}(y) d m(y)
$$

where $\varphi_{j}$ is, of course, defined by (6.6). We already know that for each fixed $t>0$ the relation (6.9) holds for almost all $x$. Thus if $t<1$ we have for all $x$

$$
\begin{aligned}
\varphi_{j}(x) & =e^{\lambda_{j}} \int k(1, x, y) \varphi_{j}(y) d m(y) \\
& =e^{\lambda_{j}} \int\left[\int k(t, x, z) k(1-t, z, y) d m(z)\right] \rho_{j}(y) d m(y) \\
& =e^{\lambda_{j} t} \int k(t, x, z) \varphi_{j}(z) d m(z),
\end{aligned}
$$

where the interchange is justified since the integral exists absolutely in the first order. If $t>1$ we have for all $x$

$$
\begin{aligned}
\int k(t, & x, y) \varphi_{j}(y) d m(y) \\
& =\int\left[\int k(1, x, z) k(t-1, z, y) d m(z)\right] \varphi_{j}(y) d m(y) \\
& =e^{\lambda_{j}(1-t)} \int k(1, x, z) \varphi_{j}(z) d m(z) \\
& =e^{-\lambda_{j} t} \varphi_{j}(x) .
\end{aligned}
$$

Thus (6.9) holds for all $t>0$ and all $x$.

For fixed $s>0$ the Schwarz inequality, (6.2), and (6.9) combine to yield

$$
\left|\varphi_{j}(x)\right|^{2} \leqq e^{2 \lambda_{j}} \int k(s, x, y)^{2} d m(y)\left\|\varphi_{j}\right\|^{2}
$$


By (6.5) we know that $\sum_{j=1}^{\infty} e^{-\lambda_{j} t}<\infty$ for all $t>0$, and combining this with (6.10) and the fact that $\left\|\varphi_{j}\right\|=1$ we obtain

$$
\sum_{j=1}^{\infty} e^{-\lambda} j^{t}\left|\varphi_{j}(x)\right|^{2}<\infty
$$

for all $t>0$ and all $x$. The Schwarz inequality and (6.11) imply that

$$
\sum_{j=1}^{\infty} e^{-\lambda_{j} t} \varphi_{j}(x) \varphi_{j}(y)
$$

converges absolutely for all $t>0$ and all $(x, y)$. Let us define

$$
h(t, x, y)=\sum_{j=1}^{\infty} e^{-\lambda_{j} t} \varphi_{j}(x) \varphi_{j}(y) .
$$

Let $t>0$ and $x$ be fixed and let $A \subset G$ with $m(A)<\infty$. Since $\int_{A}\left|\varphi_{j}(x)\right| d m(x)<[m(A)]^{1 / 2}$ it follows from (6.10) that

$$
\sum_{j=1}^{\infty} e^{-\lambda_{j} t}\left|\varphi_{j}(x)\right| \int_{A}\left|\varphi_{j}(y)\right| d m(y)<\infty .
$$

Thus by the Fubini theorem (we regard the sum as an integral over the discrete measure space $\{1,2,3, \cdots\}$ each point being assigned measure one) and (6.9)

$$
\begin{aligned}
\int_{A} h(t, x, y) d m(y) & =\sum_{j=1}^{\infty} e^{-\lambda_{j} t} \mathscr{P}_{j}(x)\left(I_{A}, \varphi_{j}\right) \\
& =\sum_{j=1}^{\infty} e^{-\lambda_{j} t / 2} \int k(t / 2, x, y) \varphi_{j}(y) d m(y)\left(I_{A}, \varphi_{j}\right) .
\end{aligned}
$$

Applying the Fubini theorem again (the integral and sum exist absolutely) we obtain

$$
\int_{A} h(t, x, y) d m(y)=\int k(t / 2, x, y) \sum_{j=1}^{\infty} e^{-\lambda_{j} t / 2}\left(I_{A}, \varphi_{j}\right) \varphi_{j}(y) d m(y) .
$$

This last sum converges absolutely but it also converges in $L_{2}(G)$ to $T_{t / 2} I_{A}$ and, of course, the $L_{2}$ sum and the pointwise sum must agree almost everywhere. Thus we finally have

$$
\begin{aligned}
& \int_{A} h(t, x, y) d m(y)=\int k(t / 2, x, y)\left(T_{t / 2} I_{A}\right)(y) d m(y) \\
= & \int_{k} k(t / 2, x, y)\left(\int_{A} k(t / 2, y, z) d m(z)\right) d m(y) \\
= & \int_{A} k(t, x, z) d m(z) .
\end{aligned}
$$

Since $A$ was an arbitrary set of finite measure it now follows that $h(t, x, \cdot)=$ $k(t, x, \cdot)$ almost everywhere for each fixed $(t, x)$, that is, $h(t, x, \cdot)$ is a 
density for $K(t, x, \cdot)$ with respect to $m$. Clearly $h(t, x, y)$ is jointly measurable in its variables and since these were the defining properties of $k$ we may (and will) set $k(t, x, y)=h(t, x, y)$ for all $t>0, x, y$ in the sequel.

\section{THEOREM 6.5. Under the assumptions of Theorem 6.4}

$$
\int k(t, x, y)^{2} d m(x) d m(y)=\sum_{j=1}^{\infty} e^{-2 \lambda_{j} t} .
$$

Proof. This is a well known property of such operators. See [12; p. 353].

7. The Spectral Decomposition:-General Case. In this section we will use the theory of generalized eigenfunction expansions to obtain a spectral decomposition for $k(t, x, y)$ without assuming $(K)$ of Theorem 6.2. We begin by giving a short outline of this theory. ${ }^{4}$

Let $T$ be a (possibly unbounded) self-adjoint operator in $L_{2}(G)$ and $E(\lambda)$ its spectral resolution. One version of the spectral theorem asserts that there exists a sequence of elements, $\left\{\mathcal{P}_{n}\right\}$, in $L_{2}(G)$ such that $L_{2}(G)$ is the orthogonal direct sum $\sum_{1}^{\infty} \mathfrak{S}_{n}$ where $\mathscr{S}_{n}$ is the closed manifold of all vectors of the form $F(T) \varphi_{n}$ where $F \in L_{2}\left(\mu_{n}\right)$ and $\mu_{n}(\cdot)=\left(E(\cdot) \varphi_{n}, \varphi_{n}\right)$. In fact the correspondence $F(T) \varphi_{n} \rightarrow F(\cdot)$ establishes an isomorphism, $U$, of $L_{2}(G)$ onto the direct sum $\Sigma=\sum_{1}^{\infty} L_{2}\left(\mu_{n}\right)$ which diagonalizes $T$ in the sense that

$$
(U F(T) \varphi)_{n}(\lambda)=F(\lambda)(U \varphi)_{n}(\lambda)
$$

for $\varphi \in \Im(F(T))$. If $\varphi \in L_{2}(G)$ then $(U \varphi)_{n}$ denotes the component of $U \varphi$ in $L_{2}\left(\mu_{n}\right)$. Moreover, each $\mathfrak{S}_{n}$ reduces $T$ and the support of each $\mu_{n}$ is contained in the spectrum of $T$. The operator $T$ is said to have a generalized eigenfunction expansion provided that there exist kernels $\mathrm{W}_{n}(\lambda, x)$ jointly measurable in $(\lambda, x)$ such that for all $\mathcal{Q} \in L_{2}(G)$

$$
(U \varphi)_{n}(\lambda)=\int \varphi(x) W_{n}^{*}(\lambda, x) d m(x)^{5}
$$

and

$$
\varphi(x)=\sum_{n=1}^{\infty} \int_{-\infty}^{\infty}(U \varphi)_{n}(\lambda) W_{n}(\lambda, x) d \mu_{n}(\lambda),
$$

where the precise meaning (7.2) and $(7.3)$ is as follows. In (7.2) there

${ }^{4}$ We follow Garding's approach to the problem [4]. A set of seminar notes entitled "Generalized Eigenfunction Expansions" giving the details of this subject may be obtained from the author upon request.

${ }^{5}$ If $\varphi$ is a complex valued function, $\varphi^{*}$ denotes its complex conjugate. 
exists an increasing sequence, $\left\{S_{k}\right\}$, of sets of finite measure such that $G=\cup S_{k}$ and $\int_{S_{k}} \varphi(x) W_{n}^{*}(\lambda, x) d m(x)$ exists as a Lebesgue integral for each $k$ and is an element of $L_{2}\left(\mu_{n}\right)$ as a function of $\lambda$. Moreover, as $k \rightarrow \infty$ this sequence of elements converges in $L_{2}\left(\mu_{n}\right)$ to the value of the integral. The intergral in (7.3) is defined similarly in $L_{2}(G)$ and the sum is then taken in $L_{2}(G)$.

A complex valued function, $b(x, y)$, defined on $G \times G$ and jointly measurable in $(x, y)$ will be called a Carleman kernel if it satisfies

$$
b(x)=\int_{G}|b(x, y)|^{2} d m(y)<\infty \text { a. e. }[m] .
$$

That is $b(x, \cdot) \in L_{2}(G)$ for almost all $x$. With the kernel, $b(x, y)$, we associate the domain, $\mathfrak{S}_{0}$, consisting of those elements $\varphi \in L_{2}(G)$ satisfying

$$
\int|\varphi(x)| b(x) d m(x)<\infty
$$

A densely defined operator, $B$, in $L_{2}(G)$ is called a Carleman operator if there exists a Carleman kernel, $b(x, y)$, such that for all $\phi \in \mathfrak{O}(B)$ we have

$$
(B \varphi)(x)=\int \varphi(y) b^{*}(x, y) d m(y) .
$$

The following theorem was proved by Gårding [4]. (Actually he proved slightly more but this is all that we will need.)

THEOREM 7.1. Let $T$ be a densely defined self-adjoint operator in $L_{2}(G)$ and we use the notation introduced above. Let $\phi$ be a complex valued function measurable with respect to all the $\mu_{n}$ and such that $|\phi|>0$ almost everywhere with respect to all the $\mu_{n}$. If $\phi(T)$ is a Carleman operator, then $T$ has a generalized eigenfunction expansion.

In proving this theorem Garding first showed that there existed functions $B_{n}(\lambda, x)$ jointly measurable in $(\lambda, x)$ such that $B_{n}(\cdot, x) \in L_{2}\left(\mu_{n}\right)$ and $B_{x}=\left\{B_{n}(\cdot, x)\right\} \in \sum$ for all $x$. If $b(x, y)$ is the Carleman kernel corresponding to $\phi(T)$ then $\left[U b(x, \cdot)\left[_{n}(\lambda)=B_{n}(\lambda, x)\right.\right.$ as elements of $L_{2}\left(\mu_{n}\right)$ for almost all $x$. Finally we have

$$
W_{n}(\lambda, x)=\phi(\lambda)^{-1} B_{n}^{*}(\lambda, x) .
$$

Garding also showed that if $\phi \in \mathfrak{O}_{0}$ then

$$
(U \varphi)_{n}(\lambda)=\int \varphi(x) W_{n}^{*}(\lambda, x) d m(x)
$$

exists as an ordinary Lebesgue integral and

$$
A_{n}(\lambda, \varphi)=\int \varphi(x) B_{n}(\lambda, x) d m(x) \in L_{2}\left(\mu_{n}\right)
$$


and $A(\varphi)=\left\{A_{n}(\cdot, \varphi)\right\} \in \sum$. Also if $F=\left\{F_{n}\right\} \in \sum$ is such that

$$
\sum_{n} \int_{-\infty}^{\infty}\left|\phi(\lambda)^{-1} F_{n}(\lambda)\right|^{2} d \mu_{n}(\lambda)<\infty
$$

then

$$
\left(U^{-1} F\right)(x)=\sum \int_{-\infty}^{\infty} F_{n}(\lambda) W_{n}(\lambda, x) d \mu_{n}(\lambda)
$$

a. e. where the integrals exist as Lebesgue integrals and sum converges absolutely. This completes our review of Gärding's theory and we now return to the problem at hand.

THEOREM $7.2 .^{6} \quad$ Let $f(t, x, y)=f(t, y, x)$ a. e. $[m \times m]$ and let $\int_{G} f(t, x, y)^{2} d m(y) \leqq b(t)<\infty$ for almost all $x$, then there exist finite measures, $\mu_{n}$, with support contained in the nonnegative reals and real kernels $W_{n}(\lambda, x)$ defined for $\lambda>0$ and $x \in G$ and jointly measurable in $(\lambda, x)$ such that

$$
h(t, x, y)=\sum_{n=1}^{\infty} \int_{0}^{\infty} e^{-\lambda t} W_{n}(\lambda, x) W_{n}(\lambda, y) d \mu_{n}(\lambda)
$$

exists for all $t, x, y$. (The integrals exists as Lebesgue integrals and the sum converges absolutely.) Moreover there exists a null set, $S$, such that for all $t>0$ and $x \notin S$ the function $h(t, x, \cdot)$ is a density for $K(V, G ; t, x, \cdot)$ with respect to $m$.

Proof. Let $T_{t}=T_{t}[V, G]$ then exactly as in Theorems 6.1 and 6.3 it follows that each $T_{t}$ is a positive definite self-adjoint operator and \| $T_{t} \|<1$. Let $\Omega_{G}^{\prime}$ be the infinitesimal generator of $\left\{T_{t} ; t \geqq 0\right\}$, then $-\Omega_{G}^{\prime}$ is a positive definite self-adjoint (but in general unbounded) operator. Moreover, if $\phi(\lambda)=e^{-\lambda}$ then $\phi\left(-\Omega_{G}^{\prime}\right)=T_{1}$. Since $k(t, x, y) \leqq$ $f(t, x, y)$ a. e. it follows that $T_{1}$ is a Carleman operator and thus $-\Omega_{G}^{\prime}$ has a generalized eigenfunction expansion. Because each $T_{t}$ and hence $-\Omega_{G}^{\prime}$ is a real operator, the isomorphism, $U$, between $L_{2}(G)$ and $\Sigma$ can be chosen to be real, i.e., $U$ commutes with complex conjugation. Thus the kernels $W_{n}(\lambda, x)$ in the generalized eigenfunction expansion of $-\Omega_{G}^{\prime}$ can be taken to be real. Since $U$ diagonalizes $-\Omega_{G}^{\prime}$ we have

$$
\left(U T_{t} \varphi\right)_{n}(\lambda)=e^{-\lambda t}(U \varphi)_{n}(\lambda)
$$

for all $\varphi \in L_{2}(G)$.

From our general discussion of Garding's theorem we know that there exists a null set, $S$, such that $(S$ independent of $n) U[k(1, x, \cdot)]_{n}(\lambda)=$ $e^{-\lambda} W_{n}(\lambda, x)$ as elements of $L_{2}\left(\mu_{n}\right)$ for $x \notin S$. But

${ }_{9}$ If $m(G)<\infty$ then the hypothesis of Theorem 7.2 implies $(\mathrm{K})$ of Theorem 6.2. Thus Theorem 7.2 contributes to our knowledge only in the case $m(G)=\infty$. If in Theorem 7.2 we assume $b(t)<\infty$ for all $x$, it then follows that $h(t, x, \cdot)$ has the required properties for all $\dot{t}$ and $x$. In this connection see, Lemma 2.2 of R. K. Getoor, An Analogue of of Mercer's Theorem, Duke Math. J. 25 (1958), 615-624. 


$$
\left[T_{t} k(s, x, \cdot)\right](y)=\int k(t, y, z) k(s, x, z) d m(z)=k(t+s, x, y)
$$

for almost all $y$ for fixed $(t, s, x)$, see (6.8).

Thus

$$
\begin{aligned}
(U k(t+1, x, \cdot)]_{n}(\lambda) & =U\left[T_{t} k(1, x, \cdot)\right]_{n}(\lambda) \\
& =e^{-\lambda t}[U k(1, x, \cdot)]_{n}(\lambda) \\
& =e^{-\lambda(t+1)} W_{n}(\lambda, x)
\end{aligned}
$$

as elements of $L_{2}\left(\mu_{n}\right)$ for $x \notin S$. On the other hand if $t<1$ and $x \notin S$ we have $e^{-\lambda} W_{n}(\lambda, x)=[U k(1, x, \cdot)]_{n}(\lambda)$

$$
\begin{aligned}
& =\left[U T_{1-t} k(t, x ; \cdot)\right]_{n}(\lambda) \\
& =e^{-\lambda(1-t)}[U k(t, x, \cdot)]_{n}(\lambda),
\end{aligned}
$$

or

$$
[U k(t, x, \cdot)]_{n}(\lambda)=e^{-\lambda t} W_{n}(\lambda, x) .
$$

Thus (7.11) holds for all $t>0$ and $x \notin S$ as elements of $L_{2}\left(\mu_{n}\right)$. If we define $W_{n}(\lambda, x)=0$ for $x \in S$ (which does not effect the generalized eigenfunction expansion) then

$$
W=\left\{e^{-\lambda t / 2} W_{n}(\lambda, x)\right\}=\left\{[U k(t / 2, x, \cdot)]_{n}(\lambda)\right\} \in \Sigma
$$

for $x \notin S$ and $W=0$ for $x \in S$ and hence

$$
\|W\|^{2}=\sum_{n=1}^{\infty} \int_{0}^{\infty} e^{-\lambda t}\left|W_{n}(\lambda, x)\right|^{2} d \mu_{n}(\lambda)<\infty
$$

for all $x$. Thus applying the Schwarz inequality twice we see that

$$
h(t, x, y)=\sum_{n=1}^{\infty} \int_{0}^{\infty} e^{-\lambda t} W_{n}(\lambda, x) W_{n}(\lambda, y) d \mu_{n}(\lambda)
$$

exists for all $t, x, y$; the integrals exist as ordinary Lebesgue integrals and the sum exists absolutely. Moreover

$$
|h(t, x, y)| \leqq h(t, x, x)^{1 / 2} h(t, y, y)^{1 / 2}
$$

We next show that $h(t, x, y)$ has the desired properties. Let $A$ be a set of finite measure, then

$$
\begin{aligned}
& \int_{A} \sum_{n=1}^{\infty} \int_{0}^{\infty} e^{-\lambda t}\left|W_{n}(\lambda, x)\right|\left|W_{n}(\lambda, y)\right| d \mu_{n}(\lambda) d m(y) \\
& \quad \leqq h(t, x, x)^{1 / 2} \int_{A} h(t, y, y)^{1 / 2} d m(y) \\
& \quad \leqq\left[m(A) h(t, x, x) \int_{A} \sum_{n=1}^{\infty} \int_{0}^{\infty} e^{-\lambda t} \mid W_{n}\left(\lambda,\left.y\right|^{2} d \mu_{n}(\lambda) d m(y)\right]^{1 / 2}\right. \\
& \quad=\left[m(A) h(t, x, x) \int_{A}\left\|\left\{e^{-\lambda t / 2} W_{n}(\lambda, y)\right\}\right\|^{2} d m(y)\right]^{1 / 2} \\
& \quad=\left[m(A) h(t, x, x) \int_{A} \int k(t / 2, y, z)^{2} d m(z) d m(y)\right]^{1 / 2} \\
& \quad \leqq[b(t / 2) h(t, x, x)]^{1 / 2} m(A)<\infty
\end{aligned}
$$


where we have used the fact that $U$ is an isomorphism between $L_{2}(G)$ and $\Sigma$. Thus we have

$$
\int_{A} h(t, x, y) d m(y)=\sum_{n=1}^{\infty} \int_{0}^{\infty} e^{-\lambda t} W_{n}(\lambda, x) \int_{A} W_{n}(\lambda, y) d m(y) d \mu_{n}(\lambda)
$$

where the interchange is easily justified by the preceding calculation and the Fubini theorem. But $I_{A} \in \mathfrak{D}_{0}$, therefore $\int_{A} W_{n}(\lambda, y) d m(y)=\left(U I_{A}\right)_{n}(\lambda)$ as elements of $L_{2}\left(\mu_{n}\right)$ and hence for all $t>0$ and all $x \in X$ we have

$$
\int_{A} h(t, x, y) d m(y)=\sum_{n=1}^{\infty} \int_{0}^{\infty} e^{-\lambda t} W_{n}(\lambda, x)\left(U I_{A}\right)_{n}(\lambda) d \mu_{n}(\lambda) .
$$

The right side of (7.12) can be written in the form $\left(\left\{e^{-\lambda t} W_{n}(\lambda, x)\right\}\right.$, $\left.\left\{\left(U I_{A}\right)_{n}(\lambda)\right\}_{\sigma}\right)_{\sigma}$ where $(\cdot, \cdot)_{\sigma}$ is the inner product in $\Sigma$. According to (7.11) there exists a null set $S$ independent of $t$ such that $e^{-\lambda t} W_{n}(\lambda, x)=$ $[U k(t, x, \cdot)]_{n}(\lambda)$ as elements of $L_{2}\left(\mu_{n}\right)$ for all $t>0$ provided $x \notin S$. Combining this with (7.12) and the fact that $U$ is an isomorphism we obtain

$$
\int_{A} h(t, x, y) d m(y)=\left(k(t, x, \cdot), I_{A}\right)=\int_{A} k(t, x, y) d m(y)
$$

for all $t>0$ and all $x \notin S$. We note that $S$ is also independent of $A$. Since (7.13) holds for all sets of finite measure the proof of Theorem 7.2 can now be completed by the standard approximation argument.

As in $\S 6$ we may as well (and do) take $k(t, x, y)=h(t . x, y)$ for all $t>0, y \in G$, and $x \notin S$. Our last theorem in this section shows that $W_{n}(\lambda, \cdot)$ is actually an eigenfunction (but not necessarily in $L_{2}$ ) of an appropriate integral equation.

THEOREM 7.3. Let the hypotheses of Theorem 7.2 hold, then for each $t>0$ and $n$ there exists a $\mu_{n}$ null set, $\Lambda_{n}$, and a $m$ null set, $S$, (both depending on $t$ in general) such that $e^{-\lambda t} W_{n}(\lambda, x)=\int l k(t, x, y) W_{n}(\lambda, y) d m(y)$ for $\lambda \notin \Lambda_{n}$ and $x \notin S$. That is, $W_{n}(\lambda, \cdot)$ is an eigenfunction of the integral equation $e^{-\lambda t} f(x)=\int f(y) k(t, x, y) d m(y)$ for almost all $\lambda$ for fixed $t>0$.

Proof. Let $b(t, x)=\int k(t, x, y)^{2} d m(y) \leqq b(t)$ for almost all $x$, then $\mathfrak{D}_{0}$ is the set of $\phi \in L_{2}(G)$ such that $\int b(1, x)|\varphi(x)| d m(x)<\infty$. Let $A$ be a set of finite measure then $I_{A} \in \mathfrak{S}_{0}$ and $T_{t} I_{A} \in \mathfrak{D}_{0}$ since

$$
\int b(1, x)\left|\left(T_{t} I_{A}\right)(x)\right| d m(x) \leqq b(1) \iint_{A} k(t, x, y) d m(y) d m(x) \leqq b(1) m(A),
$$

where we have used the symmetry of $k$. Thus for almost all $\lambda$ 


$$
\left(U T_{t} I_{A}\right)_{n}(\lambda)=\int\left(T_{t} I_{A}\right)(x) W_{n}(\lambda, x) d m(x)
$$

and

$$
\left(U T_{t} I_{A}\right)_{n}(\lambda)=e^{-\lambda t}\left(U I_{A}\right)_{n}(\lambda)=e^{-\lambda t} \int I_{A}(x) W_{n}(\lambda, x) d m(x),
$$

where the integrals are ordinary integrals.

Since the integral in (7.15) exists absolutely we can change the order of integration obtaining

$$
\left(U T_{t} I_{A}\right)_{n}(\lambda)=\int_{A} \int k(t, x, y) W_{n}(\lambda, x) d m(x) d m(y)
$$

for almost all $\lambda$. Combining this with (7.16) we see that

$$
\int_{A}\left[e^{-\lambda t} W_{n}(\lambda, y)-\int k(t, x, y) W_{n}(\lambda, x) d m(x)\right] d m(y)=0
$$

for almost all $\lambda$ where, of course, the exceptional set depends on $A$.

Using the facts that $m$ is $\sigma$-finite and $\mathfrak{B}(X)$ is countably generated it is a standard matter to conclude that for fixed $t$ there exists a $\mu_{n}$ null set, $A_{n}$, and a $m$ null set, $S$, (both depending on $t$ in general) such that $e^{-\lambda t} W_{n}(\lambda, y)=\int k(t, x, y) W_{n}(\lambda, x) d m(x)$ provided $\lambda \notin \Lambda_{n}$ and $y \notin S$. This completes the proof of Theorem 7.3.

\section{REFERENCES}

1. Austin, Blumenthal, and Chacon, On continuity of transition functions, Duke Math. Journ. 25 (1958). 533-541

2. R. M. Blumenthal, An extended Markov property, Trans. Amer. Math. Soc. 85 (1957), $52-72$.

3. J. L. Doob, Slochasic Processes, New York 1953.

4. L. Gảrding, Application of the theory of direct integrals of Hilbert. spaces to some integral and differential operators, Lecture no. 11, Institute for Fluid Dynamics and Applied Mathematics, Univ. of Maryland, 1954.

5. R. K. Getoor, Additive functionals of a Markov process, Pacific J. Math. 7 (1957), $1577-1591$.

6. E. Hille and R. S. Phillips, Functional analysis and semi-groups. Amer. Math. Soc. Colloq. Publ. Vol. 31, rev. ed. (1957).

7. G. A. Hunt, Markov processes and potentials, I, I11. Journ. of Math. 1 (1957) 44-93.

8. M. Kac, On some connections between probability theory and differential and integral equations, Proc. Second. Berk. Symp. on Math. Stat. and Prob. (1951), 189-215.

9. D. Ray, On the spectra of second order differential eperators, Trans. Amer. Math. Soc. 77 (1954), 299-321.

10. F. Riesz and B. Sz. Nagy, Leçons D'Analyse Fonctionnellc, Budapest (1953).

11. K. Yosida, On the generating parametrix of the stochastic processes, Proc. Nat. Acad. of Sci. U. S. A. 44 (1955), 240-244.

12. A. C. Zaanen, Linear Analysis, New York (1953). 


\section{PACIFIC JOURNAL OF MATHEMATICS}

\section{EDITORS}

\section{David Gilbarg}

Stanford University

Stanford, California

\section{R. A. Beaumont}

University of Washington

Seattle 5 , Washington

\author{
A. L. Whiteman
}

University of Southern California Los Angeles 7, California

L. J. Paige

University of California

Los Angeles 24, California

\author{
E. F. BECKENBACH \\ C. E. BURGESS \\ E. HEWITT \\ A. HORN
}

\author{
V. GANAPATHY IYER \\ R. D. JAMES \\ M. S. KNEBELMAN \\ L. NACHBIN
}

ASSOCIATE EDITORS
I. NIVEN

T. G. OSTROM

H. L. ROYDEN

M. M. SCHIFFER
E. G. STRAUS

G. SZEKERES

F. WOLF

K. YOSIDA

\section{SUPPORTING INSTITUTIONS}

\author{
UNIVERSITY OF BRITISH COLUMBIA \\ CALIFORNIA INSTITUTE OF TECHNOLOGY \\ UNIVERSITY OF CALIFORNIA \\ MONTANA STATE UNIVERSITY \\ UNIVERSITY OF NEVADA \\ OREGON STATE COLLEGE \\ UNIVERSITY OF OREGON \\ OSAKA UNIVERSITY \\ UNIVERSITY OF SOUTHERN CALIFORNIA
}

\author{
STANFORD UNIVERSITY \\ UNIVERSITY OF TOKYO \\ UNIVERSITY OF UTAH \\ WASHINGTON STATE COLLEGE \\ UNIVERSITY OF WASHINGTON \\ * * * \\ AMERICAN MATHEMATICAL SOCIETY \\ CALIFORNIA RESEARCH CORPORATION \\ HUGHES AIRCRAFT COMPANY \\ SPACE TECHNOLOGY LABORATORIES
}

Mathematical papers intended for publication in the Pacific Journal of Mathematics should be typewritten (double spaced), and the author should keep a complete copy. Manuscripts may be sent to any one of the four editors. All other communications to the editors should be addressed to the managing editor, L. J. Paige at the University of California, Los Angeles 24, California.

50 reprints per author of each article are furnished free of charge; additional copies may be obtained at cost in multiples of 50 .

The Pacific Journal of Mathematics is published quarterly, in March, June, September, and December. The price per volume (4 numbers) is $\$ 12.00$; single issues, $\$ 3.50$. Back numbers are available. Special price to individual faculty members of supporting institutions and to individual members of the American Mathematical Society: $\$ 4.00$ per volume; single issues, $\$ 1.25$.

Subscriptions, orders for back numbers, and changes of address should be sent to Pacific Journal of Mathematics, 2120 Oxford Street, Berkeley 4, California.

Printed at Kokusai Bunken Insatsusha (International Academic Printing Co., Ltd.), No. 6, 2-chome, Fujimi-cho, Chiyoda-ku, Tokyo, Japan.

PUBLISHED BY PACIFIC JOURNAL OF MATHEMATICS, A NON-PROFIT CORPORATION

The Supporting Institutions listed above contribute to the cost of publication of this Journal, but they are not owners or publishers and have no responsibility for its content or policies. 


\section{Pacific Journal of Mathematics}

\section{Vol. 9, No. $2 \quad$ June, 1959}

Lee William Anderson, On the breadth and co-dimension of a topological lattice

Frank W. Anderson and Robert L. Blair, Characterizations of certain lattices

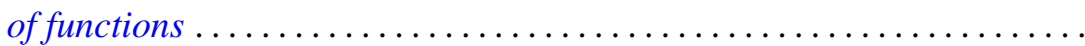

Donald Charles Benson, Extensions of a theorem of Loewner on integral

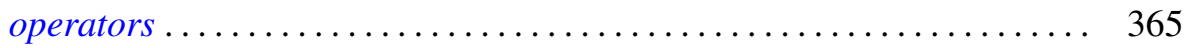

Errett Albert Bishop, A duality theorem for an arbitrary operator ........ 379

Robert McCallum Blumenthal and Ronald Kay Getoor, The asymptotic distribution of the eigenvalues for a class of Markov operators ........

Delmar L. Boyer and Elbert A. Walker, Almost locally pure Abelian

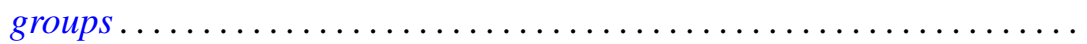

Paul Civin and Bertram Yood, Involutions on Banach algebras ........... Lincoln Kearney Durst, Exceptional real Lehmer sequences .... 415

Eldon Dyer and Allen Lowell Shields, Connectivity of topological

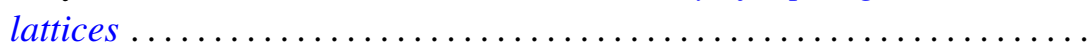

Ronald Kay Getoor, Markov operators and their associated

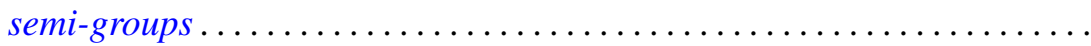

Bernard Greenspan, A bound for the orders of the components of a system of algebraic difference equations

Branko Grünbaum, On some covering and intersection properties in

Minkowski spaces ............................

Bruno Harris, Derivations of Jordan algebras ..............

Henry Berge Helson, Conjugate series in several variables.

Isidore Isaac Hirschman, Jr., A maximal problem in harmonic analysis.

II .

Alfred Horn and Robert Steinberg, Eigenvalues of the unitary part of a matrix

Edith Hirsch Luchins, On strictly semi-simple Banach algebras ...

William D. Munro, Some iterative methods for determining zeros of

functions of a complex variable...

John Rainwater, Spaces whose finest uniformity is metric .

William T. Reid, Variational aspects of generalized convex functions ....

A. Sade, Isomorphisme d'hypergroupoï des isotopes ...... . .

Isadore Manual Singer, The geometric interpretation of a special

connection . . .

Charles Andrew Swanson, Asymptotic perturbation series for characteristic



